\title{
BEAM PROPAGATION METHOD FOR LIQUID CRYSTAL-CORE OPTICAL FIBER WAVEGUIDES
}

\author{
A. SZYMAŃSKa* and T. WolińSKI \\ Institute of Physics, Warsaw University of Technology \\ Koszykowa 75, 00-662 Warszawa, Poland
}

\begin{abstract}
Propagation effects in liquid crystal-core optical fiber waveguides were investigated and simulated by means of beam propagation method. Spatial propagation beam distribution for anisotropic liquid crystal fibers was defined by using BPM_CAD software package (courtesy of Optiwave Corporation). The field intensity was related to the refractive index of core at the liquid crystal fiber in two different waveguide configurations: planar and radial, whereas the number of the propagating modes was calculated. Numerical results were compared with some experimental data formerly obtained.

PACS numbers: 42.70.Df, 42.81.Qb, 42.81.Dp
\end{abstract}

\section{Introduction}

The beam propagation method (BPM) for computing the field in an optical fiber waveguide was proposed and shown by Feit and Fleck [1-3]. Over several years this method has found many applications for different calculations.

BPM is generally applied to simulate propagation of the light beam at any power distribution in an optical fiber and then is used to determine modal characteristics of the investigated fiber. Thus, isotropic optical fibers have been studied by means of BPM as well as fibers with anisotropic core $[4,5]$, but up till now there has been no significant evidence of using BPM for liquid crystal (LC) fibers.

Over the last years there has been increasing interest in LC fibers, because of their usability in different sensors, modulators, switches, and couplers. Liquid crystal fibers owe their popularity due to the following properties:

- sensitivity to external perturbations;

- possibility of working in dangerous environments (explosive, corrosive, etc.);

- sufficient rate of data transfer;

- high level of miniaturisation.

So far beam propagation in a liquid crystal fiber has been analysed on the basis of Maxwell's equations without any approximation [5]. From the beginning [5] we calculated the Hondros-Debay equation and consequently the propagation constant for each of the modes could be found. However it was extremely difficult to achieve satisfying computer simulations and that is why we have chosen the beam propagation method.

*e-mail: agnszym@if.pw.edu.pl; http://www.if.pw.edu.pl/ opto/ 


\section{Principles of beam propagation method}

The beam propagation method consists of the recurring steps (step by step): - propagating light beam in free space;

- compensating the phase of the light beam to take into account the difference between refractive indices.

BPM introduces the following limitations: (1) small propagation step; (2) small difference between refractive indices, (3) spatial spectrum of the light beam must be limited.

\section{Theoretical analysis of light propagation in LC fibers}

The starting point of the calculation is the vector Helmholtz equation

$$
\left(\frac{\partial^{2}}{\partial x^{2}}+\frac{\partial^{2}}{\partial y^{2}}+\frac{\partial^{2}}{\partial z^{2}}\right) \boldsymbol{E}+k^{2} \boldsymbol{E}=0 \text {. }
$$

Substituting $k=\frac{n(\omega, x, y, z) \omega}{c}$, where $n(\omega, x, y, z)$ is the refractive index of the waveguide core, the Helmholtz equation (1) can be rewritten as

$$
\left(\frac{\partial^{2}}{\partial x^{2}}+\frac{\partial^{2}}{\partial y^{2}}+\frac{\partial^{2}}{\partial z^{2}}\right) \boldsymbol{E}+\frac{\omega^{2}}{c^{2}} n^{2}(\omega, x, y, z) \boldsymbol{E}=0 .
$$

The solution of Eq. (2) is postulated in the form

$$
E\left(x, y, z_{0}+\Delta z\right)=E\left(x, y, z_{0}\right) \exp (G z)
$$

where we assume $z_{0}=0$.

Substituting the solution (3) into Eq. (2) we obtain an expression of the operator $G$ ( $G$ signifies the transverse distribution of the electric field in the light beam)

$$
G=i \sqrt{\frac{\partial^{2}}{\partial x^{2}}+\frac{\partial^{2}}{\partial y^{2}}+k_{0}^{2} n^{2}(x, y, z)}
$$

and then the formula (3) yields

$$
E(x, y, \Delta z)=E(x, y, 0) \exp \left(\mathrm{i} \sqrt{\frac{\partial^{2}}{\partial x^{2}}+\frac{\partial^{2}}{\partial y^{2}}+k_{0}^{2} n^{2}(x, y, z)} z\right) .
$$

In the description of the light beam propagation in free space we accept the following substitution in Eq. (5): $n(x, y, z)=n_{\mathrm{r}}$, where $n_{\mathrm{r}}$ is the refractive index in free space. Then propagation in free space is described by Eq. (5). Taking into account expression (5) we assume

$$
E(x, y, \Delta z)=E(x, y, 0) \exp \left(\mathrm{i} \sqrt{\frac{\partial^{2}}{\partial x^{2}}+\frac{\partial^{2}}{\partial y^{2}}+k_{0}^{2} n_{\mathrm{r}}^{2}} z+\mathrm{i} k_{0}\left(n-n_{\mathrm{r}}\right) z\right)
$$

The first term in the brackets of the equation is the propagation in free space and the second is the phase compensation, respectively

$$
P_{\text {prop }}=i \sqrt{\frac{\partial^{2}}{\partial x^{2}}+\frac{\partial^{2}}{\partial y^{2}}+k_{0}^{2} n_{\mathrm{r}}^{2}}, \quad P_{\text {comp }}=\mathrm{i} k_{0}\left(n-n_{\mathrm{r}}\right) .
$$


Since the operators $P_{\text {prop }}$ and $P_{\text {comp }}$ do not commute reciprocal, therefore their . sequence of the operation influences the final result.

In case of LC-core fibers all these criteria described in Sec. 2 are satisfied and consequently beam field is transformed according to equation

$$
\begin{aligned}
& E(x, y, z+\Delta z)=E\left(x, y, z_{0}\right) \exp \left(\mathrm{i} \sqrt{\frac{\partial^{2}}{\partial x^{2}}+\frac{\partial^{2}}{\partial y^{2}}+k_{0}^{2} n_{\mathrm{r}}^{2}} \frac{\Delta z}{2}\right) \\
& \quad \times \exp \left(\mathrm{i} k_{0}\left(n-n_{\mathrm{r}}\right) \Delta z\right) \\
& \quad \times \exp \left(\mathrm{i} \sqrt{\frac{\partial^{2}}{\partial x^{2}}+\frac{\partial^{2}}{\partial y^{2}}+k_{0}^{2} n_{\mathrm{r}}^{2}} \frac{\Delta z}{2}\right) \exp \left(-\frac{x^{2}+y^{2}}{w_{0}^{2}}\right),
\end{aligned}
$$

where $\exp \left(-\frac{x^{2}+y^{2}}{w_{0}^{2}}\right)$ is a Gaussian beam and $w_{0}$ is the beam waist.

\section{Numerical simulation of light propagation in fibers}

It is assumed that a $\mathrm{He}-\mathrm{Ne}$ laser acts as a light source characterised by the Gaussian beam distribution.

Expanding $\exp (x)$ and $\sqrt{1+x}$ into series we consider only the zero- and the first-order expansions $\exp (x) \approx 1+x$, and $\sqrt{1+x} \approx 1+\frac{1}{2} x$.

Hence the following expression for $E(x, y, z+\Delta z)$ is obtained:

$$
\begin{aligned}
& E(x, y, z+\Delta z)=E_{0}\left(x, y, z_{0}\right) \exp \left(\mathrm{i} k_{0}\left(n-n_{\mathrm{r}}\right) \Delta z\right) \\
& \quad \times\left\{1+\frac{1}{2}\left[3 \mathrm{i} k_{0} n_{\mathrm{r}}-k_{0}^{2} n_{\mathrm{r}}{ }^{2} \frac{9}{8} \Delta z\right.\right. \\
& \left.\left.+\frac{\mathrm{i}}{2} \frac{\left[8(D-1)+\mathrm{i} k_{0} n_{\mathrm{r}}\left[\frac{9}{2}(D-1)+4 \frac{\left(2+D^{2}-4 D\right)}{k_{0}^{2} n_{\mathrm{r}}^{2} w_{0}^{2}}\right] \Delta z\right]}{k_{0} n_{\mathrm{r}} w_{0}^{2}}\right] \Delta z\right\} \exp (-D),
\end{aligned}
$$

where $E_{0}\left(x, y, z_{0}\right)$ is the field amplitude, $k_{0}=\frac{2 \pi}{\lambda}, \lambda$ is the wavelength, $n_{\mathrm{r}}$ is the refractive index (in general $n_{\mathrm{r}}$ is the refractive index of either the core or cladding), $n$ is the distribution of refractive index in optical fiber, $\Delta z$ is the propagation step, $D=\exp \left(-\frac{x^{2}+y^{2}}{w_{0}^{2}}\right)$ and $w_{0}$ is the waist beam.

The LC under investigation has been composed of the nematic LC core and consequently both planar and radial structures have to be considered (Fig. 1a,b). Figures ( 2 and 3 ) and calculations were obtained by using the software BPM_CAD 3.0 [6]. All of the calculations were made for the following parameters: $\lambda=$ $0.6328 \mu \mathrm{m}, r=2 \mu \mathrm{m}, n_{\mathrm{cl}}=1.4585$ and $n_{\mathrm{co}}=1.4845$ for the planar structure, and $n_{\mathrm{co}}=1.544$ for the radial structure. Figure $2 \mathrm{a}, \mathrm{b}$ and $3 \mathrm{a}, \mathrm{b}$ show the field-intensity distribution and the field intensity distribution in decibels calculated as $10 \log$ (intensity), respectively. There are bigger fluctuations of amplitude for the planar structure in Fig. 2, because there are fewer modes in this type of propagation. In the radial structure due to higher value of the refractive index $\left(n_{\mathrm{co}}=1.544\right)$ the 

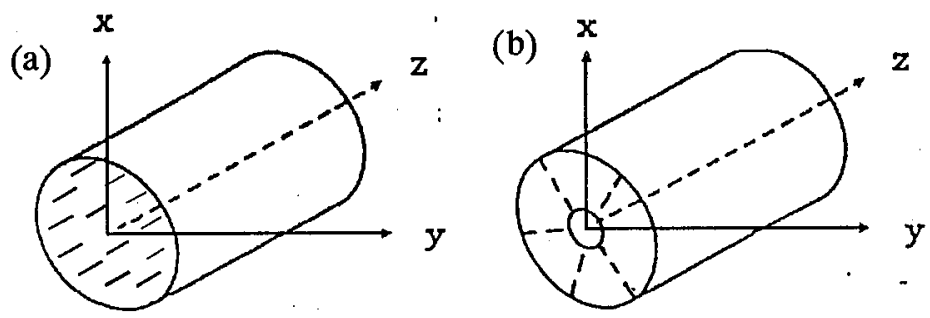

Fig. 1. Types of alignment of the director inside the liquid crystal-core fiber: (a) planar structure, (b) radial structure.

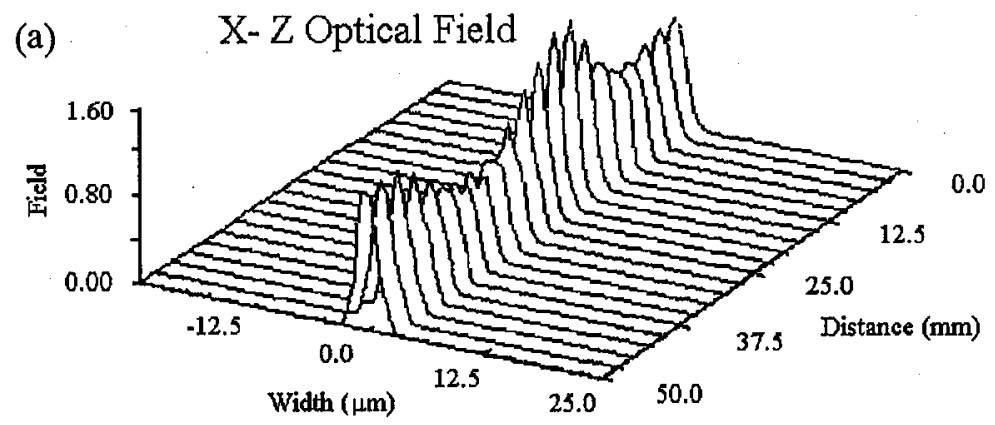

(b)

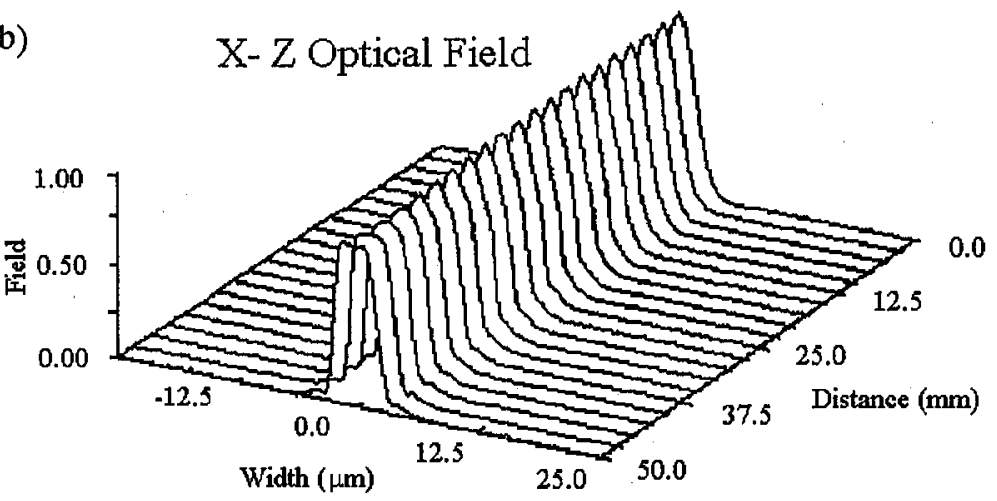

Fig. 2. Field intensity vs. length fiber for $r=2 \mu \mathrm{m}$ ( $x-z$ optical field): (a) planar structure, (b) radial structure.

modal structure of the guided field disappears. Consequently, the optical field distribution is smoothed and does not exhibit significant changes in the cross-section. Suppression is the bigger the further from the core centre.

Number of propagating modes along with their modal (effective) indices for both structures are summarised in Tables I and II. 

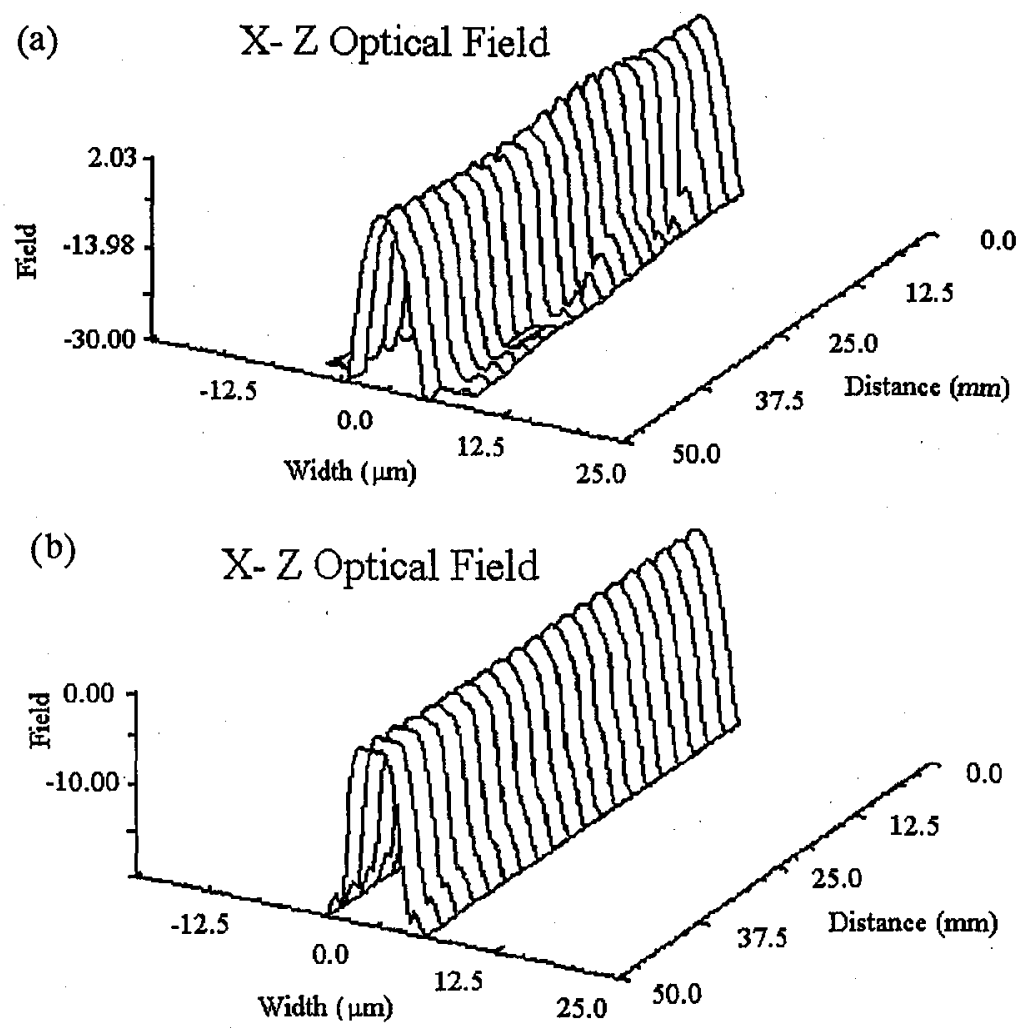

Fig. 3. Field intensity in $\mathrm{dB}$ calculated as $10 \log$ (intensity) vs. length fiber for $r=2 \mu \mathrm{m}$ $(x-z$ optical field): (a) planar structure, (b) radial structure.

TABLE I

List of modes for the

planar structure.

\begin{tabular}{c|c|c}
\hline \multicolumn{2}{l|}{} & \multicolumn{2}{c|}{$\begin{array}{c}\text { Modal } \\
\text { index }\end{array}$} \\
\hline$l$ & $p$ & index \\
\hline 0 & 1 & 1.480988 \\
0 & 2 & 1.466759 \\
1 & 1 & 1.475673 \\
2 & 1 & 1.468860 \\
3 & 1 & 1.460877
\end{tabular}

TABLE II

List of modes for the radial structure.

\begin{tabular}{|c|c|c|c|c|c|c|c|c|}
\hline \multicolumn{2}{|c|}{$\mathrm{LP}_{l p}$} & \multirow{2}{*}{$\begin{array}{l}\text { Modal } \\
\text { index }\end{array}$} & \multicolumn{2}{|c|}{$\mathrm{LP}_{l p}$} & \multirow{2}{*}{$\begin{array}{l}\text { Modal } \\
\text { index }\end{array}$} & \multicolumn{2}{|c|}{$\mathrm{LP}_{l p}$} & \multirow{2}{*}{$\begin{array}{l}\text { Modal } \\
\text { index }\end{array}$} \\
\hline$l$ & $p$ & & $l$ & $p$ & & $l$ & $p$ & \\
\hline 0 & 1 & 1.54007 & $\mathbf{I}$ & 3 & 1.47511 & 4 & 1 & 1.50485 \\
\hline 0 & 2 & 1.52333 & 2 & 1 & 1.52608 & 4 & 2 & 1.46253 \\
\hline 0 & 3 & 1.49359 & 2 & 2 & 1.49617 & 5 & 1 & 1.49167 \\
\hline 1 & 1 & 1.53403 & 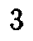 & 1 & 1.51634 & 6 & 1 & 1.47682 \\
\hline 1 & 2 & 1.51068 & 3 & 2 & 1.47999 & 7 & 1 & 1.46038 \\
\hline
\end{tabular}




\section{Conclusions}

The analysis presented above clearly indicates suitability of BPM to calculate parameters of optical fibers with the liquid crystalline core in both the radial or the planar configurations.

The formula (9) derived in the paper gives the usable tool for further computer simulations of liquid crystal fibers in particular to characterise propagation phenomena in LC fiber with any molecular ordering inside the core.

\section{Acknowledgments}

The authors are indebted to Dr. Zdzisław Jakubczyk, President of the Optiwave Corporation for the Waveguide Optics Modelling Software System BPM_CAD [6].

This work was supported by the Committee for Scientific Research under the research project no. 8T10C 04212 .

\section{References}

[1] M.D. Feit, J.A. Fleck, Appl. Opt. 17, 3990 (1978).

[2] M.D. Feit, J.A. Fleck, Appl. Opt. 18, 2843 (1979).

[3] M.D. Feit, J.A. Fleck, Appl. Opt. 19, 1154 (1980).

[4] C.L. Xu, W.P. Huang, J. Chrostowski, S.K. Chaudhuri, J. Light. Technol. 12, 1926 (1994).

[5] L. Thylen, D. Yevick, Appl. Opt. 12, 2751 (1982).

[6] BPM_CAD Waveguide Optics Modelling Software System Version 3.0, Optiwave Corporation, Nepean, Ontario, Canada. 